\title{
The multidimensional nucleus
}

\author{
This issue highlights a range of genetic techniques and cell biological models required to begin to understand the \\ levels of long-range regulation of gene expression as it occurs during cell differentiation. Explanations based on the \\ specificity of covalent modifications and binding interactions intersect with evidence for conjectured mechanisms of \\ topological loop creation and maintenance by transcription and motile protein activities.
}

$T$ he SMC family of proteins are elongated motile ATPases that can interact with naked DNA and chromatin alike. Forming rings, cohesins are capable of extruding and constraining the topology of loops, of maintaining sister chromatid cohesion and of compacting regions of the nucleus. Condensins, in contrast, play roles in mitotic chromosome condensation and have the potential to form contractile filaments, zippers or rosette structures on DNA or chromatin (reviewed by Hirano, Nat. Rev. Mol. Cell Biol. 7, 311-322, 2006, and Uhlmann, Nat. Rev. Mol. Cell Biol. 17, 399-412, 2016).

Last year, Ken-ichi Noma and colleagues (Nat. Genet. 48, 1242$1252,2016)$ demonstrated that cohesin and condensin are associated, respectively, with 50 - to $100-\mathrm{kb}$ and 100 - to $500-\mathrm{kb}$ topologically associating domains (TADs) in the fission yeast genome and can be found at the domain boundaries. Now, Frank Uhlmann and colleagues (p. 1553; see also p. 1419) have used conditional degradation of a cut14 condensin-encoding allele to identify multiple condensin-associated interphase domains that nearly double in size upon mitotic chromosome condensation in a manner that is dependent upon condensin and mitotic protein kinase activity. The roles and functions of SMC proteins in establishing topological domains in interphase chromatin in fungal, plant and mammalian genomes remain to be demonstrated.

On page 1522, Paul Khavari and colleagues examined human epidermal progenitor cells and the progressive differentiation of keratinocytes by RNA sequencing (RNA-seq) and proximity ligation (Hi-C). Nearly all differentially expressed genes (induced or repressed) over this time course fell within unchanged topological boundaries of lineage-specific TADs absent in embryonic stem cells. Transcriptional enhancer-promoter and promoter-promoter contacts typically fell within single domains of two types. Stable, cohesin-associated domains were charac- terized by preexisting H3K27 acetylation 'enhancer' chromatin marks. Enhancer-promoter contacts gained during differentiation, in contrast, acquired the H3K27 acetylation and the domains were typically not associated with cohesin.

Clusters of chromatin loops gathered with a contradictory histone mark, H3K27me3, are inhibitory to transcription in most eukaryotic contexts. On page 1546 (see also p. 1416), Doris Wagner and colleagues investigated the DNA sequence specificity of the PRC2 histone methylase complex responsible for this trimethylation in Arabidopsis. PRC2 does not contain intrinsic DNA- or chromatin-binding proteins; rather, as in Drosophila, multiple transcription factors that bind predicted PREs (Polycomb response elements) interact with PRC2 subunits, providing the complex with its sequence-specific targeting function.

Given that cells proliferate, condensed chromatin must be able to decondense and inhibitory marks need to permit reactivation. On page 1502 (see also p. 1418), Maria-Elena Torres-Padilla and colleagues used sequence-specific TALE constructs to silence or increase transcription from LINE-1 elements repeated throughout the early mouse embryonic genome. These manipulations slowed blastocyst development by interfering with chromatin accessibility and chromatin compaction, respectively, demonstrating the role of repeat transcription (although not coding or retrotransposition) in establishing the structures essential for embryonic gene expression.

Eukaryotic chromatin has many topological structures and is segmented into compartments full of highly correlated marks and protein associations (reviewed by Bonev and Cavalli, Nat. Rev. Genet. 17, 661-678, 2016). The factors and forces initiating and maintaining the multidimensional genome's functional transitions are the next frontier to fall to genetic and genomic tools. 